\title{
Cryogenic Medicinal Gas Dosage Form
}

National Cancer Institute

\section{Source}

National Cancer Institute. Cryogenic Medicinal Gas Dosage Form. NCI Thesaurus. Code C149657.

A gas which liquefies at 1.013 bar at a temperature below -150 degrees $C$. 